\title{
Retraction Note to: Ginsenoside-Rg1 enhances angiogenesis and ameliorates ventricular remodeling in a rat model of myocardial infarction
}

\author{
Huiqiu Yin • Zhaoqiang Liu • Fuhai Li • Mei Ni • \\ Bo Wang • Yun Qiao • Xinsheng Xu • Mei Zhang • \\ Jidong Zhang $\cdot$ Huixia Lu $\cdot$ Yun Zhang
}

Published online: 27 March 2013

(C) Springer-Verlag Berlin Heidelberg 2013

\section{Retraction to: J Mol Med 2011, 89:363-375 DOI 10.1007/s00109-011-0723-9}

The following article "Ginsenoside Rg1 enhances angiogenesis and ameliorates ventricular remodeling in a rat model of myocardial infarction" by Yin $\mathrm{H}$, Liu Z, Li F, Ni M, Wang B, Qiao Y, Xu X, Zhang M, Zhang J, Lu $\mathrm{H}$, Zhang Y., published in the Journal of Molecular
Medicine in Volume 89:363-375 has been retracted upon request of the authors. The retraction has been made due to duplication of blots in Figure 1a and Figure 3 in this paper. The authors claimed that this duplication was caused by their data mismanagement and they stand by the validity of their conclusions. The authors deeply apologize for any inconvenience this may have caused to the readers.

The online version of the original article can be found at:http:// dx.doi.org/10.1007/s00109-011-0723-9.

H. Yin $\cdot$ F. Li $\cdot$ M. Ni $\cdot$ X. Xu $\cdot$ M. Zhang $\cdot$ H. Lu $(\bowtie) \cdot$

Y. Zhang $(\triangle)$

The Key Laboratory of Cardiovascular Remodeling and Function Research, Chinese Ministry of Education and Chinese Ministry of Health, Qilu Hospital, Shandong University, Jinan, Shandong, China e-mail: huixialv@gmail.com

e-mail: zhangyun@sdu.edu.cn?

H. Yin $\cdot$ B. Wang $\cdot$ Y. Qiao $\cdot$ J. Zhang $(\bowtie)$

Department of Traditional Chinese Medicine, Qilu Hospital, Shandong University,

Jinan, Shandong, China

e-mail: drjidongzhang@yahoo.com.cn

\section{Z. Liu}

Department of Ophthalmology,

Provincial Hospital Affiliated to Shandong University,

Jinan, Shandong, China

F. Li

Department of Pediatrics, Qilu Hospital, Shandong University,

Jinan, Shandong, China

$\mathrm{X} . \mathrm{Xu}$

Department of Cardiology, Dongying People's Hospital,

Dongying, Shandong, China 